\title{
QUALITY OF LIFE AND REGULAR DIET IN PATIENTS WITH CHRONIC KIDNEY DISEASE
}

\author{
Svetla Staykova $^{1,2}$, Petar Petrov ${ }^{1,2}$, Lili Grudeva ${ }^{2,3}$ \\ ${ }^{1}$ Clinic of Nephrology and Dialysis, St. Marina University Hospital, Varna \\ ${ }^{2}$ Second Department of Internal diseases, Faculty of Medicine, \\ Medical University of Varna \\ ${ }^{3}$ Clinic of Hepatogastroenterology, St. Marina University Hospital, Varna
}

\begin{abstract}
INTRODUCTION: Chronic kidney disease (CKD) is characterized by a reduced rate of glomerular filtration (GF), under $60 \mathrm{~mL} / \mathrm{min} / 1.73 \mathrm{~m} 2$, and/or laboratory data, and/or image data for kidney damage, present for more than 3 months. According to recent data, nearly $8.7 \%$ of the world population suffer from CKD with different etiology. CKD is a progressive health condition that can result in an end stage renal disease (ESRD).

The quality of life $(\mathrm{QoL})$ of these patients is of utmost importance and is related to their functional activity, well-being and overall perception of their health in a physical, psychological and social aspect. A direct link between QoL, morbidity rate and death rate exists. It has been established that patients with CKD have a significantly lower QoL compared to healthy people, which becomes clearer during the more advanced stages of the disease. Physical activity decreases progressively with the progression of kidney disease.

RESULTS: Sociodemographic, clinical and laboratory risk factors have been established in the population of dialysis patients, which definitely leads to a change in QoL. Malnutrition, which is observed in patients with CKD, can also contribute to a decline in their quality of life. Its occurrence results from a reduced food intake, increased protein consumption and altered endocrine function of the kidneys. Oral intake of nutrients raises the chances of treating protein-energy wasting (PEW) in patients with ESRD.
\end{abstract}

CONCLUSION: Conducting dietary consultations, as well as preparing individual diets to meet a patient's specific needs, will increase their quality of life.

Keywords: chronic kidney disease, protein energy waste, quality of life, hemodialysis, peritoneal dialysis

Address for correspondence:

Petar Petrov

Clinic of Nephrology and Dialysis

1 Hristo Smirnenski Blvd

St. Marina University Hospital

9000 Varna

e-mail:petar_ppv@yahoo.com

Received: August 15, 2019

Accepted: September 4, 2019

\section{INTRODUCTION}

Malnutrition is a major characteristic of chronic kidney disease (CKD), which emerges in varying degrees depending on the stage of the disease. In between $40 \%$ and $70 \%$ of the patients who are experiencing end stage renal disease (ESRD), malnutrition is discovered as a result of various factors that include loss of appetite, digestion and nutrient assimilation disorder, metabolic acidosis, and emotional stress (1). 
The quality of life (QoL) for these patients is important and related to their functional activity, wellbeing, and general perception of their health in a physical, psychological and social aspect $(2,3)$. There is a direct link between QoL, morbidity rate, and death rate. Malnutrition that is observed in peritoneal dialysis patients contributes significantly to the reduced QoL they lead $(4,5,6)$.

\section{Protein-Energy Wasting (PEW)}

Protein-energy wasting (PEW) is a term suggested by the International Society of Renal Nutrition and Metabolism (ISRNM). It includes numerous nutritional and catabolic changes, which appear during a chronic kidney disorder, and is linked to an increased morbidity and death rate. Insufficient food intake (the actual malnutrition), due to a lack of appetite and food restrictions, contributes to its occurrence and results from reduced food import, increased protein consumption, and a change in the endocrine function of the kidneys. Changes caused by uremia are also accountable, such as increased energy consumption, persistent inflammation, acidosis, and numerous metabolic disorders $(7,8)$. They, in turn, lead to a state of hypermetabolism that causes excess catabolism of muscle tissue and fat deposits. For patients with chronic kidney disease, poor physical activity, weakness, as well as the dialysis itself, additionally contribute to PEW. Research using classic markers for nutritional status shows that nearly 18 $75 \%$ of the patients with CKD, who are subjected to chronic dialysis treatment, show indications of protein-energy malnutrition or malnutrition syndrome - cachexia.

Protein energy waste is a state of reduced protein and energy resources (body proteins and fats). This anomaly is often linked with reduced functional capacity and metabolic load. Protein or energy depletion could be a result of wrong diet, while in kidney diseases, conditions/syndromes, which lead to weight loss unrelated to reduced nutrients intake, have been discovered (9). These are unusual inflammatory processes, transient and intercurrent catabolic diseases, nutrient loss during dialysis procedures, endocrine disorders (resistance to insulin, growth hormone and insulin-like growth factor-1 (IGF-1), hyperglucagonemia, hyperparathyroidism), as well as blood loss in the hemodialysis system (10).
Baltzan and Shoker think that at least two types of malnutrition could appear in dialysis patients. The first type (type 1) is itself associated with the uremic syndrome or with factors related to uremia, such as reduced physical activity, ineffective dialysis procedure, dietary restrictions, and psychosocial factors. Baltzan and Shocker et al., in 1998, report on moderate reduction in the levels of serum albumin on account of reduced intake of protein and energy, taking into consideration uremic toxicity, which causes uremic anorexia with respective reduction in protein catabolism $(11,12)$. On the other hand, a more distinctive hypoalbuminemia, the significantly increased oxidation stress, as well as the increased protein catabolism, characterize the other type of malnutrition (type 2). Significant comorbid states, such as chronic heart failure and infectious complications, are common with this type of malnutrition, which can be proved through higher levels of C-reactive protein (CRP) and anti-inflammatory cytokines (13).

\section{Reasons for Protein-Energy Wasting (PEW)}

1. Reduced protein intake: anorexia, dysregulation in the circulating mediators connected with appetite, uremic toxins, dietary restrictions, changes in the organs (participating in the nutrients intake), depression, inability to take in or prepare food;

2. Hypermetabolism: increased energy consumption (inflammation), increased circulating antiinflammatory cytokines, higher levels of CRP;

3. Secondary factors: related to obesity, insulin resistance, hormone disorders (insulin resistance in CKD, increased glucocorticoid activity);

4. Metabolic acidosis;

5. Reduced physical activity;

6. Reduced anabolism: reduced nutrient intake, resistance to growth hormone/IGF-1, shortage of testosterone, lower levels of the thyroid gland hormones, accompanying diseases (diabetes, heart failure, etc.), and lower quality of life;

7. Dialysis: loss of nutrients during the dialysis procedure - dialysis membranes, hemodialysis lines, dialysis solutions, hypermetabolic processes connected to the loss of remaining kidney function. 


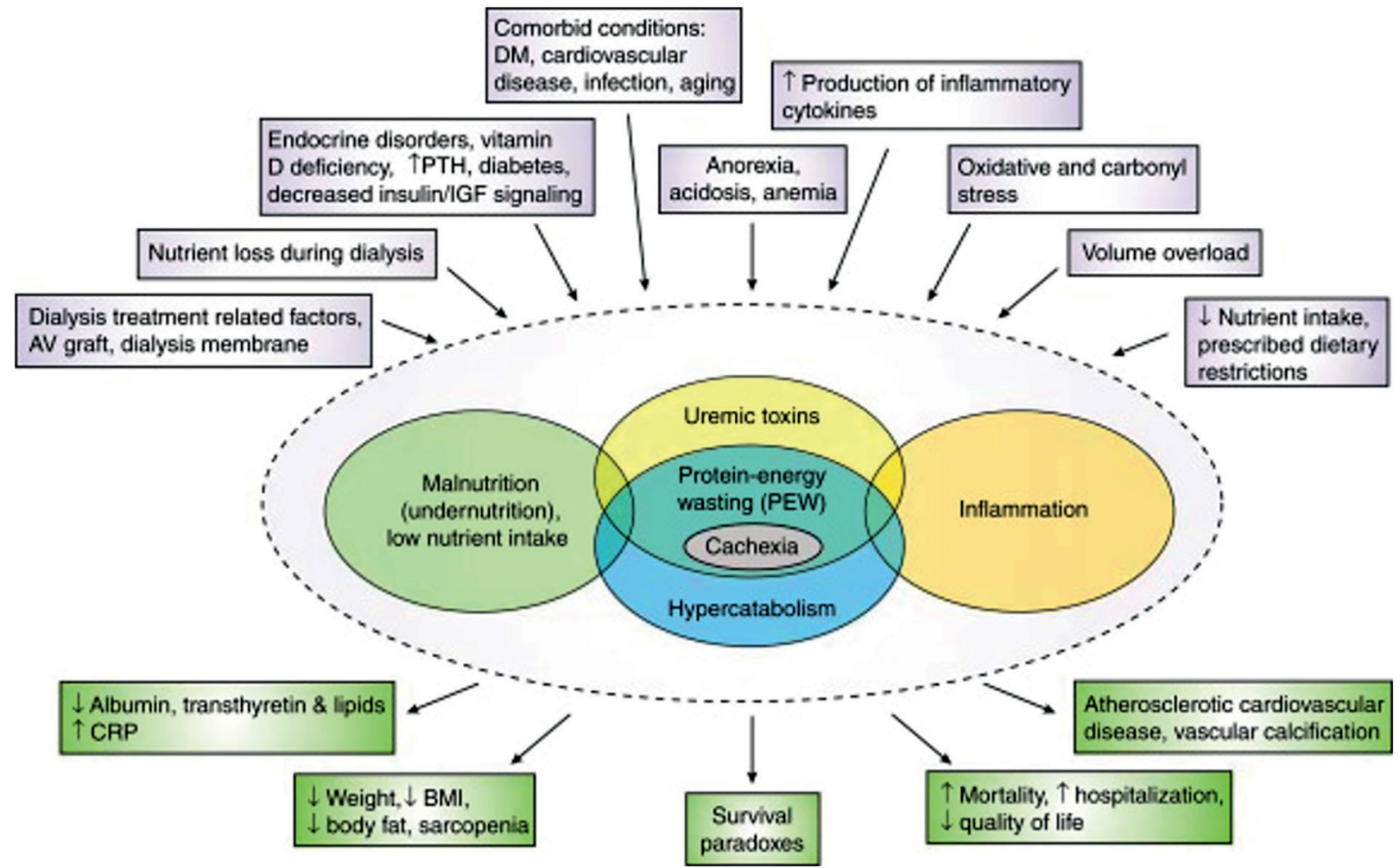

Fig. 1. Schematic presentation of the causes and emergence of protein energy malnutrition in kidney diseases (14)

\section{MATERIALS AND METHODS}

Markers tracking quality of life are presented in this article.

A regular diet plays an important role in reducing the morbidity rate and death rate for patients with CKD. The recommendations given by the Kidney Disease Improving Global Outcomes (KDIGO) (2012) suggest that pre-dialysis patients with CKD G 4-5 should go on a protein diet of $0.8 \mathrm{~g} / \mathrm{kg} / \mathrm{day}$, which ensures a quality diet. A diet consisting of a lower intake of proteins is useful when controlling hyperphosphatemia, metabolic acidosis, and hyperkaliemia. Protein restriction could prevent CKD from progressing by hemodynamically mediated decrease in inner glomerular pressure, as well as from changes in the expression of cytokines. The hemodynamic effects of the protein-induced hyperfiltration result from changes in the hormones (such as glucagon and IGF-1), changes in the renin-angiotensin system and intrarenal effects, including tubuloglomerular feedback $(14,15)$.

On the other hand, for patients with advanced CKD who are subjected to a hemodialysis treatment the protein intake has to be $1.5 \mathrm{~g} / \mathrm{kg} / \mathrm{day}$, while for patients who are subjected to a continuous ambulatory peritoneal dialysis the protein intake is about $2 \mathrm{~g} / \mathrm{kg} /$ day. It is important to note that the protein has to have high biological value (BV). Hemodialysis patients lose considerable amounts of amino acids during the procedure itself, and these losses are even higher for patients who are subjected to a continuous ambulatory peritoneal dialysis (CAPD). A suitable and adequate dialysis dose (dialysis adequacy) significantly prevents the catabolism of proteins.

\section{Hypoalbuminemia}

Hypoalbuminemia is an important marker of malnutrition, increased death rate and higher morbidity for patients undergoing kidney-replacement treatment. It has been established that a low level of serum albumin is an independent predictor of general and cardiovascular death for patients subjected to CAPD. In a number of studies and analysis, it has been proved that the levels of serum albumin could be low even in visibly well-nourished hemodialysis patients. The concentration of serum albumin is regulated by a number of factors - by protein malnutri- 
tion mostly, inflammation and external losses. The latter is important, particularly for patients subjected to continuous dialysis, where owing to the transperitoneal processes, the protein losses could reach 20 $\mathrm{g} / 24 \mathrm{~h}$. The inflammation causes hypoalbuminemia by suppressing the albumin synthesis as well as by transferring albumin from the vessel to the extravascular space. The combination of inflammation and reduced protein intake could lead to a significant decrease in the concentration of serum albumin. The pro-inflammatory cytokines also cause malnutrition and cardiovascular diseases. This is not unexpected as serum albumin and CRP participate in the same process during the acute stage of the inflammation. It has been determined that moderately increased plasma concentrations of CRP are connected to a higher risk of cardiovascular diseases for healthy individuals. Increased levels of CRP correlate with cardiovascular morbidity rate, ischemic brain insult, and other causes for elderly people. A direct link between malnutrition, increased levels of CRP, and atherosclerosis for dialysis patients has been proved.

Human plasma consists of various antioxidant biomolecules, and what is of interest is serum albumin, which is a major active antioxidant in the plasma. In this way, its low levels, which are related to malnutrition and/or inflammation, could increase the atherogenesis by increasing oxidation stress. In 1999, Soejima et al. showed that hypoalbuminemia accelerates lipid peroxidation of the erythrocyte membrane for hemodialysis patients. The mechanism through which higher oxidation stress could accelerate atherogenesis is by affecting the endothelium function (16).

\section{Atherosclerosis}

Atherosclerosis is connected with endothelium dysfunction and reduced biological presence of nitrogen oxide (NO). Both peritoneal dialysis patients and hemodialysis patients have impaired endothelium vasodilatation. The formation of NO, along with serum albumin, has relaxing properties regarding the endothelium. In this way, low levels of albumin could be associated with impaired endothelium-dependent vasodilatation. In 1999, Kin et al. demonstrate correlations between serum albumin, CRP and serum markers for endothelium function, and prove that albumin infusion does not normalize endothe- lium function. Therefore, their discoveries firmly indicate that the connection between low levels of serum albumin and endothelium dysfunction could be secondary with reference to other factors. In 1999, Kessler et al. report that in rabbits the pro-inflammatory mediators inhibit the formation hyperpolarization factor dependent on endothelium, which can contribute to endothelium dysfunction (17).

Hypoalbuminemia and inflammation are important predictors of death in dialysis patients, while malnutrition complications for them are a common cause of morbidity and hospitalization. It is established that malnutrition constitutes less than $5 \%$ of deaths in patients with CKD, while atherosclerotic cardiovascular disease is the most common cause of mortality in the dialysis population. This is the reason for the presence of a syndrome consisting of malnutrition, inflammation and atherosclerosis (MIA syndrome) in some patients with CKD. Inflammation is more common in malnourished hemodialysis patients, while in 2013, Ikizler et al. show that the nutrition status and the inflammation response are independent predictors of hospitalization of these patients. All of this shows that nutrition and inflammation markers are closely connected with cardiovascular diseases in CKD. Therefore, increased levels of pro-inflammatory cytokines are the connection between the high circulation of the inflammation, malnutrition and cardiovascular diseases in patients.

\section{CRP and Pro-Inflammatory Cytokines}

Serum levels of CRP reflect the generation of pro-inflammatory cytokines [interleukin-1, (IL-1), interleukin-6, (IL-6) and tumor necrosis factor- $\boldsymbol{\alpha}$ (TNF- $\boldsymbol{\alpha})$ ], which are reported to be increasing in patients with CKD. High levels of pro-inflammatory cytokines could cause muscle loss by stimulating protein catabolism, by decreasing albumin synthesis as well as by inhibiting appetite. In addition, it has been observed that IL-1, TNF- $\boldsymbol{\alpha}$, and endotoxins could induce catabolism of muscle protein by stimulating ketoacidotic dehydrogenase, which leads to higher oxidation of amino acids. As a result, the increased plasma levels of pro-inflammatory cytokines predict hypoalbuminemia and death in patients who are subjected to a replacement treatment of the kidney function. 
CRP is a marker convenient to use in daily practices with regard to the evaluation of the inflammatory status of patients with CKD (18). Each rise in CRP by $1 \mathrm{mg} / \mathrm{L}$ leads to $30 \mathrm{kcal}$ of daily energy consumption. Not only is the inflammation catabolic, but it is also responsible for anorexia. For dialysis patients, the increase in serum CRP is inversely connected to appetite. In 2008, K. Zadeh et al. report of an inverse connection between appetite and serum CRP in 331 patients $(19,20)$.

\section{Visfatin}

Visfatin is a new factor generated by adipocytes, also sensitive to inflammation, which could play a part in anorexia in patients with CKD. Based on the responses to an appetite questionnaire given by 246 hemodialysis patients in Sweden, high-level visfatin (e.g. over $40 \mathrm{ng} / \mathrm{mL}$ ) is linked to reduced appetite and lower plasma amino acid profile.

\section{Oxidation Stress}

Oxidation stress, which occurs during the presence of excess production of free radicals or low levels of antioxidant, is an important factor that contributes to the development of endothelium dysfunction and atherogenesis. Patients with malnutrition in stage G3-G4 of CKD have lower plasma levels of vitamin E, which suggests insufficient intake of antioxidants when the food intake is low (21). According to recent data, inflammation is connected with an increase in oxidation stress as a result of activating monocytes in patients with CKD. Because of that, it could be argued that the increase in oxidation stress contributes to a higher occurrence of cardiovascular diseases in malnourished patients with CKD.

\section{RESULTS / DISCUSSION}

Quality of life (QoL) is related to a patient's functional activity, their wellbeing and overall perception of health in a physical, psychological and social aspect. In chronic diseases, more particularly in CKD, there is a close link between QoL, morbidity rate, and mortality rate. It has been established that patients with CKD have a significantly lower QoL compared to healthy people, which is more distinct during the pre-dialysis stages (stages G4 and G5) (22). A patient's age is also of importance, especially for those in advanced age (23).

In their publication from 2011, Maria C. Cruz et al. report that QoL is reduced in all stages of CKD.
Physical activity decreases progressively in all stages of kidney disease. Malnourished patients have a significantly lower level of physical functionality compared to well rehabilitated patients. This shows that malnutrition in the early stages of CKD leads to a reduced ability to perform daily physical needs, as well as walking. The symptoms and the severity of kidney diseases are statistically and/or clinically more distinct in malnourished patients, which leads to lower QoL (24). Patients with malnutrition report of a higher influence of uremic symptoms, such as muscle weakness and pain, headaches, spasms, skin irritation, dyspnea, dizziness, lack of appetite, excessive thirst, limb numbness, memory problems, dim eyesight, nausea, etc. Sociodemographic, clinical and laboratory risk factors have been determined in the dialysis population (level of education, sex, personal approach, professional activity, age, levels of hemoglobin, serum levels of phosphorus, diabetes and accompanying diseases), which could also contribute to an altered QoL. Some of the variables (e.g. age, sex, and ethnicity) are difficult and slow to impact. It is necessary to put effort into reducing the influence of those factors, which could positively affect a patient's condition, such as treating anemia (increasing the levels of hemoglobin), and adequate treatment of accompanying diseases (25).

Both nutrition status and inflammatory response are independent predictors leading to a rise in the number of hospitalizations of patients with CKD. CRP values, serum albumin, pre-albumin, as well as the remaining inflammation factors, are reliable and important indictors of forecasting an impending disease. The mortality and morbidity rates for patients with ESRD are high.

\section{CONCLUSION}

According to researches, conducted so far, the lifespan of patients with ESRD in the USA is between 20 and 25 years shorter than the average for the population in the USA for the age group of over 45 . The connection between malnutrition, inflammation, and QoL in patients with CKD is clearly established. The provision of good food status and adequate treatment could lead to the improvement and maintenance of good QoL. Through personalized, welltimed and appropriate therapy, as well as through the prevention of symptoms caused by reduced kid- 
ney function, the progression of the disease could be slowed down significantly. For these patients, prevention and early diagnosis constitute the prevention of progression, complications, and unfavorable outcome of CKD.

\section{REFERENCES}

1. Sahay M, Sahay R, Baruah MP, Pradesh A. Nutrition in chronic kidney disease. J Med Nutr Nutraceut. $2014 ; 3(1): 11-8$.

2. Neto JF, Ferraz MB, Cendoroglo M, Draibe S, Yu L, Sesso R. Quality of life at the initiation of maintenance dialysis treatment--a comparison between the SF-36 and the KDQ questionnaires. Qual Life Res. 2000;9(1):101-7. doi:10.1023/A:1008918609281.

3. Condé SA, Fernandes N, Santos FR, Chouab A, Mota MM, Bastos MG. Cognitive decline, depression and quality of life in patients at different stages of chronic kidney disease. J Bras Nefrol. 2010;32(3):242-8.

4. Stenvinkel P, Heimbürger O, Lindholm B, Kaysen GA, Bergström J. Are there two types of malnutrition in chronic renal failure? Evidence for relationships between malnutrition, inflammation and atherosclerosis (MIA syndrome). Nephrol Dial Transplant. 2000;15(7):953-60. doi:10.1093/ ndt/15.7.953.

5. Carrero JJ1, Stenvinkel P, Cuppari L, Ikizler TA, Kalantar-Zadeh K, Kaysen G, et al. Etiology of the protein-energy wasting syndrome in chronic kidney disease: a consensus statement from the International Society of Renal Nutrition and Metabolism (ISRNM). J Ren Nutr. 2013;23(2):77-90. doi: 10.1053/j.jrn.2013.01.001.

6. Stenvinkel $\mathrm{P}$, Chung $\mathrm{SH}$, Heimbürger $\mathrm{O}$, Lindholm B. Malnutrition, inflammation, and atherosclerosis in peritoneal dialysis patients. Perit Dial Int. 2001;21 Suppl 3:S157-62.

7. Fassett RG, Venuthurupalli SK, Gobe GC, Coombes JS, Cooper MA, Hoy WE. Biomarkers in chronic kidney disease: a review. Kidney Int. 2011 Oct;80(8):806-21. doi: 10.1038/ki.2011.198.

8. Kim SB, Chi HS, Park JS, Hong CD, Yang WS. Effect of increasing serum albumin on plasma D-dimer, von Willebrand factor, and platelet aggregation in CAPD patients. Am J Kidney Dis. 1999; 33(2):312-7. doi: 10.1016/s0272-6386(99)70306-9.

9. Mehrotra, R, Kopple J. Causes of protein-energy malnutrition in chronic renal failure. In: Kopple J,
Massry S, editors Nutritional management of renal disease. 2nd ed. Philadelphia: Lippincott Williams \& Wilkins; 2003.

10. Campbell KL, Ash S, Bauer JD. The impact of nutrition intervention on quality of life in pre-dialysis chronic kidney disease patients. Clin Nutr. 2008;27(4):537-44. doi: 10.1016/j.clnu.2008.05.002.

11. Pecoits-Filho R, Lindholm B, Stenvinkel P. The malnutrition, inflammation, and atherosclerosis (MIA) syndrome-the heart of the matter. Nephrol Dial Transplant. 2002;17 Suppl 11:28-31. doi: 10.1093/ndt/17.suppl_11.28.

12. Baltzan MA, Shoker AS. Malnutrition and dialysis (letter). Kidney Int. 1998; 54(3):999.

13. Kaysen GA, Eiserich, JP. The role of oxidative stress-altered lipoprotein structure and function and microinflammation on cardiovascular risk in patients with minor renal dysfunction. J Am Soc Nephrol. 2004; 15:538-48.

14. Fouque D, Kalantar-Zadeh K, Kopple J, Cano N, Chauveau P, Cuppari L, et al. A proposed nomenclature and diagnostic criteria for protein-energy wasting in acute and chronic kidney disease. Kidney Int. 2008;74(3):393, doi: 10.1038/sj.ki.5002585.

15. Fouque D, Pelletier S, Mafra D, Chauveau P. Nutrition and chronic kidney disease, Kidney Int. 2011;80(4):348-57. doi: 10.1038/ki.2011.118.

16. Soejima A, Matsuzawa N, Miyake N, Karube M, Fukuoka K, Nakabayashi K, et al. Hypoalbuminemia accelerates erythrocyte membrane lipid peroxidation in chronic hemodialysis patients. Clin Nephrol. 1999; 51(2):92-7.

17. Kessler P, Popp R, Busse R, Schini-Kerth VB. Proinflammatory mediators chronically downregulate the formation of the endothelium-derived hyperpolarizing factor in arteries via a nitric oxide/ cyclic GMP-dependent mechanism. Circulation. 1999;99(14):1878-84. doi:10.1161/01.CIR.99.14.1878.

18. Owen WF, Lowrie EG. C-reactive protein as an outcome predictor for maintance hemodialysis patient. Kidney Int. 1998; 54(2): 627-36. doi: 10.1046/j.1523-1755.1998.00032.x.

19. Kalantar-Zadeh K, Block G, McAllister, CJ, Humphreys $\mathrm{MH}$, Kopple JD. Appetite and inflammation, nutrition, anemia and clinical outcome in hemodialysis patients. Am J Clin Nutr. 2004; 80(2):299-307.

20. Kalantar-Zadeh K, Kilpatrick RD, Kuwae N, McAllister CJ, Alcorn H Jr, Kopple JD, et al. Revisiting 
mortality predictability of serum albumin in the dialysis population: time dependency, longitudinal changes and population-attributable fraction. Nephrol Dial Transplant. 2005; 20(9):1880-8. doi: 10.1093/ndt/gfh941.

21. Kalender B, Ozdemir AC, Dervisoglu E, Ozdemir O. Quality of life in chronic kidney disease: effects of treatment modality, depression, malnutrition and inflammation. Int J Clin Pract. 2007;61(4):56976. doi: 10.1111/j.1742-1241.2006.01251.x.

22. Spiegel BM, Melmed G, Robbins S, Esrailian E. Biomarkers and health-related quality of life in endstage renal disease: a systematic review. Clin J Am Soc Nephrol. 2008;3(6):1759-68. doi: 10.2215/ CJN.00820208.

23. Herselman M, Moosa MR, Kotze TJ, Kritzinger M, Wuister S, Mostert D. Protein-energy malnutrition as a risk factor for increased morbidity in long-term hemodialysis patients. J Ren Nutr. 2000; 10(1):7-15. doi: 10.1016/s1051-2276(00)90017-7.
24. Mingardi G, Cornalba L, Cortinovis E, Ruggiata R, Mosconi P, Apolone G. Health-related quality of life in dialysis patients. A report from an Italian study using the SF-36 Health Survey. DIA-QOL Group. Nephrol Dial Transplant. 1999;14(6):150310. doi: 10.1093/ndt/14.6.1503.

25. Cruz MC1, Andrade C, Urrutia M, Draibe S, Nogueira-Martins LA, Sesso Rde C. Quality of life in patients with chronic kidney disease. Clinics (Sao Paulo). 2011;66(6):991-5. doi: 10.1590/ s1807-59322011000600012. 